\title{
LINflow: A computational pipeline that combines an alignment-free with an alignment-based method to accelerate generation of similarity matrices for prokaryotic genomes
}

\author{
Long Tian $^{\text {Equal first author, } 1 \text {, Reza Mazloom }}{ }^{\text {Equal first author, } 2}{ }^{\text {, Lenwood S Heath }}{ }^{2}$, Boris A Vinatzer ${ }^{\text {Corresp. } 1}$ \\ ${ }^{1}$ School of Plant and Environmental Sciences, Virginia Tech, Blacksburg, VA, United States \\ 2 Department of Computer Science, Virginia Polytechnic Institute and State University (Virginia Tech), Blacksburg, VA, United States \\ Corresponding Author: Boris A Vinatzer \\ Email address: vinatzer@vt.edu
}

Background Computing genomic similarity between strains is a prerequisite for genome-based prokaryotic classification and identification. Genomic similarity was first computed as Average Nucleotide Identity (ANI) values based on the alignment of genomic fragments. Since this is computationally expensive, faster and computationally cheaper alignment-free methods have been developed to estimate ANI. However, these methods do not reach the level of accuracy of alignment-based methods.

Methods Here we introduce LINflow, a computational pipeline that infers pairwise genomic similarity in a set of genomes. LINflow takes advantage of the speed of the alignment-free sourmash tool to identify the genome in a dataset that is most similar to a query genome and the precision of the alignment-based pyani software to precisely compute ANI between the query genome and the most similar genome identified by sourmash. This is repeated for each new genome that is added to a dataset. The sequentially computed ANI values are stored as Life Identification Numbers (LINs), which are then used to infer all other pairwise ANI values in the set. We tested LINflow on four sets, 484 genomes in total, and compared the needed time and the generated similarity matrices with other tools.

Results LINflow is up to 150 times faster than pyani and pairwise ANI values generated by LINflow are highly correlated with those computed by pyani. However, because LINflow infers most pairwise ANI values instead of computing them directly, ANI values occasionally depart from the ANI values computed by pyani. In conclusion, LINflow is a fast and memory-efficient pipeline to infer similarity among a large set of prokaryotic genomes. Its ability to quickly add new genome sequences to an already computed similarity matrix makes LINflow particularly useful for projects when new genome sequences need to be regularly added to an existing dataset. 
1 LINflow: A Computational Pipeline that Combines an Alignment-free with an

2 Alignment-based Method to Accelerate Generation of Similarity Matrices for

3 Prokaryotic Genomes

4

5 Long Tian ${ }^{1 *}$, Reza Mazloom², Lenwood S. Heath ${ }^{2}$, Boris A. Vinatzer ${ }^{1}$

$6{ }^{1}$ School of Plant and Environmental Sciences, Virginia Tech, Blacksburg, VA, USA

$7 \quad{ }^{2}$ Department of Computer Science, Virginia Tech, Blacksburg, VA, USA

8 Corresponding author:

9 Boris A. Vinatzer, Latham Hall, room 551, Blacksburg, VA 24061, email: vinatzer@vt.edu

$10 *$ equal contribution

11 


\section{Background}

14 Computing genomic similarity between strains is a prerequisite for genome-based prokaryotic

15 classification and identification. Genomic similarity was first computed as Average Nucleotide Identity

16 (ANI) values based on the alignment of genomic fragments. Since this is computationally expensive,

17 faster and computationally cheaper alignment-free methods have been developed to estimate ANI.

18 However, these methods do not reach the level of accuracy of alignment-based methods.

\section{Methods}

20 Here we introduce LINflow, a computational pipeline that infers pairwise genomic similarity in a set of

21 genomes. LINflow takes advantage of the speed of the alignment-free sourmash tool to identify the

22 genome in a dataset that is most similar to a query genome and the precision of the alignment-based

23 pyani software to precisely compute ANI between the query genome and the most similar genome

24 identified by sourmash. This is repeated for each new genome that is added to a dataset. The

25 sequentially computed ANI values are stored as Life Identification Numbers (LINs), which are then used

26 to infer all other pairwise ANI values in the set. We tested LINflow on four sets, 484 genomes in total,

27 and compared the needed time and the generated similarity matrices with other tools.

\section{Results}

29 LINflow is up to 150 times faster than pyani and pairwise ANI values generated by LINflow are highly correlated with those computed by pyani. However, because LINflow infers most pairwise ANI values

31 instead of computing them directly, ANI values occasionally depart from the ANI values computed by

32 pyani. In conclusion, LINflow is a fast and memory-efficient pipeline to infer similarity among a large set

33 of prokaryotic genomes. Its ability to quickly add new genome sequences to an already computed

34 similarity matrix makes LINflow particularly useful for projects when new genome sequences need to be 35 regularly added to an existing dataset. 
37 LINflow is available at https://code.vt.edu/linbaseproject/LINflow/

38

Peer) reviewing PDF | (2020:07:51115:2:0:NEW 11 Jan 2021) 
The number of prokaryotic genome assemblies available at the National Center for Biotechnology

41 Institute (NCBI) is growing rapidly and has reached 615,000 in 2020 . It can be anticipated that many

42 more genome assemblies will be published in the near future because of continued improvements in next-generation DNA sequencing technologies concerning throughput and sequence quality and a concomitant drop in sequencing cost. The ever-growing collection of prokaryotic genomes provides the opportunity to explore evolutionary relationships among species, genomic boundaries of species, and the genetic diversity within species.

DNA-DNA hybridization (DDH) was the first method that incorporated genome content in prokaryotic classification and became the gold standard in the 1970s (Brenner 1973). Two strains that have a reciprocal DDH value of over $70 \%$ are considered to belong to the same species (Brenner 1973). However, the low resolution, laborious experimental procedures, and limited portability of results present serious limitations (Stackebrandt \& Goebel 1994). After the advent of next-generation sequencing, DDH has largely been replaced by Average Nucleotide Identity (ANI). ANI is a measure of similarity between two genomes based on the comparison of whole genome sequences (Konstantinidis \& Tiedje 2005a). In its original implementation, one genome is used as a query genome and is cut into consecutive $1020 \mathrm{nt}$-long fragments. Each fragment is then aligned to the second genome, the subject genome, using BLAST (Konstantinidis \& Tiedje 2005a). Alignments that result in over $30 \%$ coverage and $70 \%$ identity are retained and $\mathrm{ANI}$ is computed as the average identity of these alignments. An ANI between $95 \%$ and $96 \%$ has been found to correspond to $70 \%$ DDH (Goris et al. 2007). ANI even provides the resolution necessary to separate strains into different genome similarity groups within species 60 (Rodriguez-R et al. 2018; Vinatzer et al. 2016). While computing ANI between a single pair of genome sequences using the alignment-based

62 BLAST algorithm is reasonably efficient, computing all pairwise ANI values for thousands of genomes 
63 (thus requiring up to millions of pairwise comparisons) is slow. Therefore, various methods have been

64 developed to infer ANI based on alignment-free algorithms. In 2015, Ondov and colleagues published

65 the first implementation of MinHash (Broder 1997) for prokaryotic genome comparisons, Mash (Ondov

66 et al. 2016). Mash and similar tools, such as sourmash (Brown \& Irber 2016; Pierce et al. 2019), produce

67 a reduced representation of a genome as a sketch (also referred to as a signature). This is done by

68 determining the presence of all k-mers in a genome sequence and using a hash function to translate

69 these k-mers into hashes, of which a subset is used as the sketch. Mash and sourmash then compare

70 genomes by calculating the Jaccard similarity between their sketches (Ondov et al. 2016). This results in

71 an estimate of the Jaccard similarity between the entire k-mer sets of the two genomes. Not only was it

72 possible with this approach to process and calculate the pairwise similarity of 54,118 microbial genomes

73 from NCBI RefSeq release 70 in 33 CPU hours, but this approximate Jaccard similarity also correlates

74 with ANI almost linearly for ANI values from approximately $90 \%$ to $99 \%$. Therefore, Mash and sourmash

75 can be used to precisely and quickly cluster genomes into species.

76 The $k$ value, i.e., the k-mer length employed when a sketch is made, significantly impacts the

77 computed Jaccard similarity. A smaller $k$ value enables the detection of similarity between genomes of

78 distantly related strains but loses resolution when the ANI between genomes is high. On the other hand,

79 longer k-mers provide high resolution when ANI is high but they cannot detect any similarity between

80 genomes of more distantly related strains (Brown \& Irber 2016; Pierce et al. 2019).

81 FastANI is another tool to determine how similar genome sequences are to each other (Jain et

82 al. 2018b; Jain et al. 2018c). However, instead of building a sketch of a whole-genome sequence, FastANI

83 maintains the conceptual framework of BLAST-based ANI: it breaks the query genome into non-

84 overlapping fragments and only in the next step replaces BLAST with an alignment-free k-mer approach,

85 called MashMAP (Jain et al. 2018a). FastANI is 50 to 4000 times faster than BLAST-based ANI, while

86 inferring ANI accurately for ANI values as low as $80 \%$ (Jain et al. 2018b; Jain et al. 2018c).

Peer] reviewing PDF | (2020:07:51115:2:0:NEW 11 Jan 2021) 
The Life Identification Number (LIN) system is a genome similarity-based system to classify

88 individual organisms based on reciprocal ANI (Marakeby et al. 2014; Vinatzer et al. 2017; Vinatzer et al.

89 2016; Weisberg et al. 2015). A LIN consists of a series of positions, where each position indicates an ANI

90 threshold, from low to high, starting from the leftmost position. The LIN of a genome is assigned based

91 on the ANI to its most similar genome whose LIN has been already assigned. Therefore, the more similar

92 two genomes are to each other, the longer their LINs are identical starting from the leftmost position. A

93 group of strains sharing the same leading part of LINs is called a LINgroup, denoted by the shared part of

94 their LINs. It has been shown that LINgroups can be used to circumscribe groups of prokaryotes from the

95 genus level to the intraspecies level, almost reaching outbreak resolution (Vinatzer et al. 2016).

To analyze the diversity of a collection of prokaryotic genomes, computing all pairwise

comparisons cannot be avoided by any of the above methods and their implementations. However,

98 when dealing with a large number of genomes, pairwise comparisons are computationally expensive.

99 Furthermore, because of the ever-growing number of sequenced genomes and their frequent addition

100 to existing datasets, re-analysis of datasets each time new genomes are added becomes necessary.

101 Here we alleviate the bottleneck of pairwise ANI computations by developing LINflow, a pipeline

102 that efficiently constructs highly resolved similarity matrices from $70 \%$ to $99.9 \%$ ANI by combining the

103 speed of the MinHash-based sourmash tool (Brown \& Irber 2016; Pierce et al. 2019) with the precision

104 of the BLAST-based pyani tool (Pritchard 2014) and the LIN concept (Weisberg et al. 2015). The obtained

105 results can then provide the basis for genome-based classification of prokaryotes. 


\section{METHODS}

\section{Overview}

109 In short, to minimize the number of computationally expensive ANI computations when constructing a

110 genomic similarity matrix, LINflow sequentially adds genomes to a dataset, at each step efficiently

111 identifies the genome already in the dataset that is most similar to the newly added genome, precisely

112 calculates the ANI value only between this pair of genomes, and assigns a LIN to the new genome based

113 on this ANI value and the LIN of the most similar genome. The LINs are then used to further accelerate

114 the identification of the most similar genome and, most importantly, to efficiently infer all remaining

115 pairwise ANI values to construct the complete genome similarity matrix. In other words, the main

116 purpose of LINs in LINflow is to reduce the number of necessary ANI computations to one per genome

117 when computing a genome similarity matrix.

118 The LINflow flowchart is shown in Figure 1. When a new genome is added to the dataset,

119 LINflow identifies the genome that is most similar to this new genome among the genomes that were

120 previously added using the computationally efficient alignment-free tool sourmash (Brown \& Irber 2016;

121 Pierce et al. 2019). This is accomplished via a two-step procedure, which consists of first identifying the

122 LINgroup to which the new genome belongs and then identifying the most similar genome in this

123 LINgroup. By default, LINflow uses the 95\%-level LINgroup in this step, but this can be modified by the

124 user based on the expected genomic similarities in a specific dataset. The precise ANI value between the

125 two genomes is then computed using the more computationally expensive, but precise, pyani tool

126 (Pritchard 2014). LINflow uses this ANI value to assign a LIN to the new genome based on the LIN of its

127 most similar genome (which LIN was previously assigned based on the ANI value to its most similar

128 genome when that genome itself was previously added to the dataset). The assigned LINs can then be 
129 used to infer all-against-all ANI values even though only a single ANI computation is performed for each 130 genome.

131 To make the results reusable and easily accessible in terms of reading and writing, a relational

132 database managed by MySQL (MySQL) is used to store data, with the schema shown in Figure 2. This

133 relational database connects tables with primary and foreign keys, and the connections between tables

134 are represented by arrows. The genome table stores the locations of the genome sequences. The

135 taxonomy table stores the taxonomic information corresponding to each genome in the database. LIN

136 schemes (i.e., the number of LIN positions and the corresponding ANI thresholds), based on which LINs

137 are assigned, are kept in the Scheme table. Besides three default LIN schemes, new schemes can be

138 added by users so that LINs can be assigned according to the users' needs in resolution. LINs are

139 assigned with the three default schemes and with one user-defined scheme if there is any.

140 The individual steps of the pipeline are described in detail below.

Generation and storing of signatures

142 LINflow uses sourmash version 2.0 (Pierce et al. 2019) to generate signatures for both $k=21$ and $k=51$

143 with $\mathrm{n}=2000$ (i.e., each signature consists of 2000 hashes) for all genomes and stores them as individual

144 files. The first member of each 95\%-level LINgroup is chosen as the representative genome and a copy of

145 its signature file is saved in a second directory together with signature files of all other representative 146 genomes.

\section{Choice of LIN scheme}

148 LINflow allows the user to choose from four default LIN schemes: (1) a 20-position LIN scheme that

149 ranges from $70 \%$ ANI to $99.999 \%$ ANI to cope with genus to strain level differentiation and is currently

150 used in LINbase (Tian et al. 2020) (Table 1), (2) a 300-position scheme with positions starting at 70\% and

151 increasing by 0.1 , and reaching $99.9 \%$ (used in this manuscript with the datasets listed below), (3) a 
1523000 -position scheme starting at $70 \%$ and reaching $99.99 \%$ at $0.01 \%$ intervals (recommended for

153 constructing highly resolved similarity matrices for strains belonging to the same species), (4) a 300,000-

154 position scheme starting at $70 \%$ and reaching $99.99 \%$ at $0.00001 \%$ intervals. The user can also define

155 any custom LIN scheme with any number of positions using any ANI value of choice (however, it is not

156 advised to use ANI values below $70 \%$ since ANI does not reflect evolutionary relationships when ANI falls

157 below $70 \%)$.

Initiation of LINflow

159 LINflow, by default, includes the 20-position LIN scheme as one of the schemes during each run. An

160 arbitrary genome will be selected to assign the first LIN with 0 in each position. Its signature will be

161 generated and saved as the representative of the LINgroup $0_{A} 0_{B} 0_{C} O_{D} O_{E} O_{F}$ using the 20-position default

162 scheme both in the directories for representative genomes and this LINgroup.

\section{LIN assignment}

164 The new genome's $\left(G_{\text {Query }}\right)$ signature $S_{\text {Query }}$ will be first queried against the representative genomes of all than 6000 bacterial genomes from different families with $k=21$, the Jaccard similarity of 0.2475 was found to correspond to $95 \%$ ANI (data not shown). If the highest Jaccard similarity $J$ to one of the representative genomes $S_{\text {Query }}$ is above 0.2475 , then the corresponding genome represents the 95\%-

170 similar to $G_{\text {Query, }}$ which means they share at least the A position in the default LIN scheme. If $J<=0.0025$,

171 the corresponding genome is less than $70 \%$ similar to $G_{\text {Query. }}$

173 most similar genome $G_{\text {subject }}$ according to Jaccard similarity in $L_{95 \%}$ is identified as the most similar

174 genome to $G_{\text {Query }}$ in the entire database. 
176 with $\mathrm{k}=21$. The most similar genome $G_{\text {Subject }}$ according to Jaccard similarity in $L_{95 \%}$ is identified as the 177 most similar genome to $G_{\text {Query }}$ in the entire database.

178 For the above cases, $A N I$ between $G_{\text {Query, }}$ and $G_{\text {subject }}, A N I_{\text {Query }}$, will be calculated with pyani. To

179 assign the LIN to the query genome, $G_{\text {subject }}$ 's LIN, $L I N_{\text {subject, }}$ will be used as the reference from A to the

180 last position that the ANI threshold is lower than or equal to $A N I_{\text {Query, }}$ the first position that ANI

181 threshold is larger than $A N I_{\text {Query }}$ will be assigned a number that has not been used with the prefix in the 182 database, and the rest of the positions will be filled with 0s. For example, $A N I_{\text {Query }}=95.4575 \%$, it is over

$18395 \%$ at the $\mathrm{F}$ position but lower than $96 \%$ at the $\mathrm{G}$ position, so it will use $\operatorname{LIN}_{\text {subject }}$ from A to $\mathrm{F}$ as $L I N_{\text {Query }}$ 'S

184 A to F. At $L I N_{\text {Query }}$ 's G position, a number that has never been used together with $L_{I} N_{\text {Subject }}$ 's prefix from A 185 to $\mathrm{G}$ will be assigned. Each of $L I N_{\text {Query }}$ 's $\mathrm{H}$ to $\mathrm{T}$ positions will be assigned 0.

186 If $J \leq 0.0025$, no genome in the current database has over $70 \%$ ANI to $G_{\text {Query, }}$ so that a new 187 number that has never been used in the A position before will be assigned to $L I N_{\text {Query's }}$ A position, and 188 the rest of $L I N_{\text {Query }}$ will be filled with Os.

\section{Update of database and signature file system}

$G_{\text {Query }}, G_{\text {Subject }} A N I_{\text {Query, }}$ and $L I N_{\text {Query }}$ will all be written to the database. If $L I N_{\text {Query }}$ creates a new $95 \%$-level

191 LINgroup, a new directory for this LINgroup will be created and $S_{\text {Query }}$ will be saved in this directory as a

192 member and as a representative genome with other representative genomes, otherwise, $S_{\text {Query }}$ will be 193 only saved in the existing 95\%-level LINgroup it belongs to.

\section{Datasets}


198 unnamed and undescribed Pseudomonas species. Dataset B consists of 43 Xanthomonas perforans

199 genomes. Dataset C includes 140 genomes of the genus Lysinibacillus, whereby 96 of them belong to 27

200 named species. Dataset D includes 53 genomes from the species Xylella fastidiosa and two genomes

201 from the species Xylella taiwanensis. A separate dataset E with the genome sequence of Pseudomonas

202 caeni DSM 24390 was used to assess the computational speed of the above tools when adding a new

203 genome to the already-analyzed dataset A. Supplementary Table 1 lists the genomes included in each

204 dataset and the actual genome sequences can be accessed directly in this repository:

205 https://code.vt.edu/linbaseproject/linflow datasets.

\section{Comparison of tools}

LINflow was compared with pyani (blast option), sourmash, and FastANI in regard to speed,

memory usage, and accuracy. Parameters used when running these programs are listed in Table 2. Note

ANI of B to A [8]. We used the average of the two pairwise ANI values. The calculations were executed

on a $2.4 \mathrm{GHz}$ CPU on Cascades, an Advanced Research Computing (ARC) system at Virginia Tech, and the execution time and memory usage were monitored by the job scheduler built in the system.

214 of each dataset calculated by the four software suites using custom R scripts. Heatmaps were generated

215 based on the hierarchical clustering results to investigate whether FastANI, LINflow and Sourmash are

216 able to classify bacteria into species as accurate as pyani. Rows and columns of the heatmaps were

217 reordered to be in the same order as the heatmap generated by pyani.

218 Finally, pairwise Mantel tests (Mantel 1967) in combination with Pearson correlation

219 coefficients were performed using custom R scripts to determine how well the similarity matrices (ANI

220 for LINflow, FastANI, and pyani and Jaccard similarity for sourmash) obtained with the different tools. 


\section{RESULTS}

\section{Computational speed and memory usage}

224 The CPU time needed to analyze datasets A, B, C, and D by each software is shown in Table 3. For sourmash, two separate times are indicated since sourmash runs two separate commands: "sourmash similarity between signatures. Although the FastANI workflow is also split into two phases, the indexing phase and the compute phase, the two phases cannot be executed separately. Therefore, only the total execution time is shown.

Sourmash was the fastest out of the four tools, being 1000-4000 times faster than pyani,

84-253 times and 20-150 times faster, respectively.

The memory usage of LINflow was lower than pyani and lower than FastANI when analyzing

datasets $A$ and $C$, which have relatively larger numbers of genomes compared to datasets $B$ and D (Table

4). Furthermore, the time cost for adding a new genome to an existing dataset by LINflow did not a new genome to dataset $\mathrm{A}$ (Table 5) with the average processing time for each genome in dataset $\mathrm{A}$

238 (Table 3).

\section{Accuracy}

240 Similarity matrices obtained with sourmash, FastANI, and LINflow were compared to those obtained

241 with pyani, which we considered the gold standard, since it is exclusively based on BLAST reflecting the

242 original description of ANI (Konstantinidis \& Tiedje 2005a; Konstantinidis \& Tiedje 2005b). For sourmash,

243 we determined its performance separately for $k=21$ and $k=51$. For LINflow, we used two of the four

244 default schemes: the 20-position scheme used in LINbase (Tian et al. 2020) (see Table 1 for the LIN 
245 scheme and Supplementary Table 1 for the result) and the 300-position scheme (with ANI values

246 increasing from $70 \%$ at the left-most LIN position to $99.9 \%$ at the right-most LIN position with $0.1 \%$

247 intervals between neighboring positions). The LINbase scheme was used to assign LINs to the genomes

248 and classify them as LINgroups. The 300-position scheme was used to determine the ANI similarity

249 matrix. After similarity matrices were computed with all tools, heatmaps were generated to visualize the

250 genomic relatedness among the analyzed genomes.

Heatmaps derived from the ANI matrices obtained with pyani (Figure 3A), FastANI (Figure 3B),

252 and LINflow (Figure 3C) show the same species level (ANI $\geq 95 \%$ ) clustering of the 247 Pseudomonas

253 genomes of dataset A visible as red blocks along the diagonal. Five major clusters are easily visible.

254 Cluster 1 consists of genomes belonging to $P$. aeruginosa, cluster 2 represents the species $P$.

255 chlororaphis, clusters 3,4 , and 5 constitute the $P$. syringae species complex and related genomes. Note

256 that LINflow not only classified the genomes as species but also distinguished intraspecific groups as

257 LINgroups. The LIN prefixes denote LINgroups and show both intergroup and intragroup relationships.

Sourmash was able to perform species-level clustering with $k$ values of both 21 (Figure 3D) and

51 (Figure 3E). The obtained results suggest that Jaccard similarity calculated with $k=51$ only weakly

correlates with ANI for low ANI values compared to $k=21$, for example, clusters 2, 4 and 5 . Instead of

resolution than $\mathrm{k}=21$.

To further evaluate the accuracy of LINflow compared to the other tools, the complete similarity

264 matrices obtained for all four datasets with all tools were compared with each other using the Mantel

265 test (Mantel 1967) using Pearson's correlation coefficients. Results are reported in Figure 4. One can

266 easily see how the results obtained with LINflow are highly correlated with those obtained with pyani

267 for datasets $A, C$, and D (Pearson correlation coefficient of 0.99 or 1 ) but not for dataset B, which has a

268 Pearson correlation coefficient of only 0.78 . Since many pairs of genomes in set B have ANI values above 
$26999.8 \%$ and differ from each other by less than $0.1 \%$, we computed ANI values for set B also using the

270 3,000-position and a 300,000-position LIN scheme hypothesizing that the lower correlation was due to

271 rounding of ANI values to the first decimal place when using the 300-position LIN scheme. However,

272 switching to the 3,000-position and 300,000-position LIN scheme only increased the correlation

273 between LINflow and pyani slightly to a Pearson correlation coefficient of 0.82 .

274 When comparing Pearson Correlation coefficients for the FastANI versus pyani comparison and

275 the sourmash versus pyani comparison with the LINflow versus pyani comparison, LINflow shows the

276 same or higher correlation with pyani for all datasets with the exception of dataset B, for which LINflow

277 shows the lowest correlation with pyani.

278 Finally, to determine how LINflow and FastANI correlate with pyani from low to high pairwise

279 ANI values, we plotted all pairwise ANI results obtained with LINflow and FastANI against pyani results

280 similar to what was done by Jain et al. (2018c). Figure 5 shows how FastANI and LINflow both correlate

281 very well with pyani for ANI values above $85 \%$ but deviate from pyani at ANI values from $85 \%$ to $70 \%$.

282 While ANI values computed with FastANI are higher than the corresponding pyani ANI values in this

283 range, ANI values inferred by LINflow merge into a relatively small number of ANI values for many

284 different pyani ANI values (see Discussion for an explanation of this phenomenon).

285

286

DISCUSSION

287 Here we developed a new tool, LINflow, to efficiently compute genome similarity matrices for genome-

288 based classification of prokaryotes. We compared the performance of LINflow with that of sourmash,

289 FastANI, and pyani when analyzing four sets of genomes and when adding a new genome to an already-

290 analyzed dataset.

291 The execution time to compare each new genome to a growing dataset does not increase as

292 significantly for LINflow as for the other tools. This is because, for each genome, the LINflow algorithm

Peer] reviewing PDF | (2020:07:51115:2:0:NEW 11 Jan 2021) 
293 involves only a one-time sourmash signature generation, at most two sourmash signature comparisons,

294 and a one-time two-way ANI calculation with pyani between the new genome and its most similar

295 genome identified by sourmash. We thus expect that, with larger datasets, LINflow will outperform

296 FastANI in terms of speed and memory usage and that the relative increase in speed compared with

297 pyani will be even more significant.

298 The LIN approach had been shown previously to correlate well with core genome phylogenetic trees within the genus Pseudomonas (Vinatzer et al. 2016). Previous comparisons of FastANI (Jain et al. 2018c), sourmash (Pierce et al. 2019), and pyani (Pritchard 2014) focused on accuracy in assigning

301 strains to species around the 95\% ANI species threshold. Here we performed a species level comparison 302 and found LINflow to perform similarly well to sourmash, FastANI, and pyani (Figure 3). However, we 303 then went on and compared the relative performance of all four tools in creating complete similarity 304 matrices from around $70 \%$ ANI to almost $100 \%$ ANI. We did this comparison using the Mantel test 305 (Mantel 1967) and computing Pearson correlation coefficients for all pairwise tool comparisons, similar

306 to what was done by Jain et al. (2018c), when comparing ANI values obtained by FastANI with ANI values

307 obtained by BLAST. LINflow had the better correlation with pyani compared to sourmash and fastANI

308 except for dataset B, which is composed of highly similar genomes. Even changing the LIN scheme to the

309300,000 -position LIN scheme did not improve performance of LINflow showing that this was

310 independent of the resolution of the deployed LIN scheme. Moreover, for pairwise ANI values below

$31185 \%$, we noticed how ANI values computed by LINflow for many different pairs of genomes merged into

312 a few identical ANI values. This is a direct result of the way LINflow infers ANI values based on

313 sequentially assigned LINs. For example, if a hypothetical group of genomes (group 1) contains genomes

314 that are all over $99 \%$ similar to each other and all genomes in a second group of genomes (group 2) are

315 all over $99 \%$ similar to each other but the first genome in group 1 that was assigned a LIN and the first

316 genome in group 2 that was assigned a LIN have a pairwise ANI value of only $70.75 \%$, then all pairwise 
317 ANI values between group 1 and group 2 genomes will be inferred to be $70.75 \%$ by LINflow. Because

318 LINs are assigned sequentially, this is an inherent limitation of LINflow that users need to weigh against

319 the time savings LINflow provides compared to pyani or FastANI.

320 The comparison between tools also revealed that FastANI's performance is affected by genomic

321 diversity of the analyzed datasets. In fact, FastANI's correlation with pyani was relatively low for

322 datasets $\mathrm{A}$ and $\mathrm{C}$, where for each dataset, the genomes are from different species; FastANI's correlation

323 with pyani was high for datasets $B$ and $D$, where most of the genomes for each dataset are from the

324 same species.

325 Finally, LINflow stores data in a MySQL relational database that organizes genomic data and the 326 corresponding metadata. MySQL is a relational database that can be accessed from its command-line 327 interface, various application programming interfaces, and graphical user interfaces. Therefore, users

328 can easily retrieve genome sequences for other analyses, e.g., comparative genomics or customized 329 reference databases, by querying the database with filters of taxonomic information and/or LINs. CONCLUSIONS

332 LINflow is a fast and memory-efficient pipeline to infer similarity among a large set of prokaryotic

333 genomes and achieves accuracy that approximates, but does not reach, that of pyani. Its ability to 334 quickly add new genome sequences to an already computed similarity matrix makes LINflow particularly 335 useful for projects when new genome sequences need to be regularly added to an existing dataset.

336 Further improvements to LINflow in regard to speed and resolution are underway. 
339 The authors acknowledge Advanced Research Computing (ARC) at Virginia Tech for providing

340 computational resources and technical support that have contributed to the results reported within this

341 paper.

342

343

REFERENCES

BRENNER DJ. 1973. Deoxyribonucleic Acid Reassociation in the Taxonomy of Enteric Bacteria. International Journal of Systematic and Evolutionary Microbiology 23:298-307. https://doi.org/10.1099/00207713-23-4-298

347 Broder AZ. 1997. On the resemblance and containment of documents. Proceedings Compression and Complexity of SEQUENCES 1997 (Cat No97TB100171). p 21-29.

Brown CT, and Irber L. 2016. sourmash: a library for MinHash scketching of DNA. Journal of Open Source Software 1:27. doi: 10.21105/joss.00027

Galili T. 2015. dendextend: an R package for visualizing, adjusting and comparing trees of hierarchical clustering. Bioinformatics (Oxford, England) 31:3718-3720. 10.1093/bioinformatics/btv428

Goris J, Konstantinidis KT, Klappenbach JA, Coenye T, Vandamme P, and Tiedje JM. 2007. DNA-DNA hybridization values and their relationship to whole-genome sequence similarities. International

Jain C, Dilthey A, Koren S, Aluru S, and Phillippy AM. 2018a. A Fast Approximate Algorithm for Mapping Long Reads to Large Reference Databases. Journal of computational biology : a journal of computational molecular cell biology 25:766-779. 10.1089/cmb.2018.0036 10.1093/bioinformatics/bty597 
362 Jain C, Rodriguez-R LM, Phillippy AM, Konstantinidis KT, and Aluru S. 2018c. High throughput ANI

363 analysis of 90K prokaryotic genomes reveals clear species boundaries. Nature Communications 9:5114. 10.1038/s41467-018-07641-9

Konstantinidis KT, and Tiedje JM. 2005a. Genomic insights that advance the species definition for prokaryotes. 102:2567-2572.

Konstantinidis KT, and Tiedje JM. 2005b. Towards a Genome-Based Taxonomy for Prokaryotes. 187:6258-6264. 10.1128/JB.187.18.6258

Mantel N. 1967. The detection of disease clustering and a generalized regression approach. Cancer research, 27(2), 209-220

Marakeby H, Badr E, Torkey H, Song Y, Leman S, Monteil CL, Heath LS, and Vinatzer BA. 2014. A System to Automatically Classify and Name Any Individual Genome-Sequenced Organism Independently of Current Biological Classification and Nomenclature. PLOS ONE 9:e89142.

MySQL. https://dev.mysql.com/

376 Ondov BD, Treangen TJ, Melsted P, Mallonee AB, Bergman NH, Koren S, and Phillippy AM. 2016. Mash: fast genome and metagenome distance estimation using MinHash. Genome biology 17:132-132. 10.1186/s13059-016-0997-x

Pierce N, Irber L, Reiter T, Brooks P, and Brown C. 2019. Large-scale sequence comparisons with sourmash [version 1; peer review: 2 approved]. F1000Research 8. 10.12688/f1000research.19675.1

Pritchard L. 2014. pyani: Python module for average nucleotide identity analyses. https://github.com/widdowquinn/pyani.

Rodriguez-R LM, Gunturu S, Harvey WT, Rosselló-Mora R, Tiedje JM, Cole JR, and Konstantinidis KT. 
Archaea and Bacteria at the whole genome level. Nucleic Acids Res 46:W282-W288.

387 10.1093/nar/gky467

Sokal RR, and Rohlf FJ. 1962. The Comparison of Dendrograms by Objective Methods. Taxon 11:33-40. $10.2307 / 1217208$

Stackebrandt E, and Goebel BM. 1994. Taxonomic Note : A Place for DNA-DNA Reassociation and s rRNA Sequence Analysis in the Present Species Definition in Bacteriology.846-849.

392

Tian L, Huang C, Mazloom R, Heath LS, and Vinatzer BA. 2020. LINbase: a web server for genome-based

Vinatzer BA, Tian L, and Heath LS. 2017. A proposal for a portal to make earth's microbial diversity easily accessible and searchable. Antonie van Leeuwenhoek 110:1271-1279. 10.1007/s10482-017-

Vinatzer BA, Weisberg AJ, Monteil CL, Elmarakeby HA, Sheppard SK, and Heath LS. 2016. A Proposal for a Genome Similarity-Based Taxonomy for Plant-Pathogenic Bacteria that Is Sufficiently Precise to Reflect Phylogeny, Host Range, and Outbreak Affiliation Applied to Pseudomonas syringae sensu Assigned to Ebolavirus Genomes Are Informative of Species Membership , Associated Outbreaks

\section{FIGURE LEGENDS}

407 Figure 1. Workflow of LINflow. The flowchart of the LIN assignment algorithm used in LINflow. 408 
409 Figure 2. Database schema used by LINflow. The relational database connects tables with primary and

410 foreign keys. Connections between tables are represented by arrows.

411

412 Figure 3. Similarity matrices obtained by LINflow, FastANI, sourmash and pyani. Heatmaps based on

413 hierarchical clustering using the complete linkage method using the similarity matrices obtained with

414 pyani (A), FastANI (B), LINflow (C), Sourmash k=21 (D), and k=51 (E) for dataset A. Cluster 1 corresponds

415 to $P$. aeruginosa, cluster 2 represents $P$. chlororaphis, clusters 3,4 , and 5 are different phylogroups within

416 the $P$. syringae species complex and related genomes. The same figure showing strain names is included

417 as Supplementary Figures 1 through 5 (corresponding to panels A through E). See Supplementary Figures

4186 through 20 for heatmaps of datasets B, C, and D.

419

420 Figure 4. Comparison of similarity matrices obtained by LINflow, FastANI, sourmash and pyani.

421 Heatmaps showing Pearson correlation coefficients based on the Mantel test performed between the 422 similarity matrices obtained with pyANI, sourmash, FastANI, and LINflow for datasets A, B, C, and D.

424 Figure 5. Correlation between the ANI results obtained with LINflow and FastANI and the ANI results obtained with pyani (using the BLAST option) for datasets A through D. A Plot showing the ANI values computed by LINflow (in blue) and FastANI (in red) on the Y axis for ANI results obtained with pyani (X axis) for all pairwise genome comparisons in datasets A through D. Pearson correlation coefficients for FastANI versus pyani and LINflow versus pyani results and all other tool comparison results are shown in compared to the pyani ANI values for all pairwise ANI values for datasets A though D. 


\section{Table $\mathbf{1}$ (on next page)}

The primary LIN assignment scheme of LINbase used to assign LINs by LINflow in this study. 
1

\begin{tabular}{|c|c|c|c|c|c|c|c|c|c|c|c|c|c|c|c|c|c|c|c|c|}
\hline $\bar{z}$ & ㅇ & $\stackrel{n}{N}$ & ঃ & $\stackrel{\llcorner}{\infty}$ & ৪ & นั & ด̆ & б̆ & ळ & $\stackrel{\text { ஸn }}{\infty}$ & ন & $\stackrel{\text { ஸे }}{\text { مे }}$ & ஸ் & $\begin{array}{l}\text { 号 } \\
\text { बं }\end{array}$ & बே & $\begin{array}{l}\stackrel{\text { N }}{\text { }} \\
\text { }\end{array}$ & $\begin{array}{l}\text { ผุ } \\
\text { ू̆ }\end{array}$ & $\begin{array}{l}\text { ஸ̆ } \\
\text { ুே }\end{array}$ & $\begin{array}{l}\text { बे } \\
\text { ুே }\end{array}$ & $\begin{array}{l}\text { ஜे } \\
\text { ஜே }\end{array}$ \\
\hline $\begin{array}{c}\text { Positi } \\
\text { on }\end{array}$ & $A$ & $B$ & $C$ & $\mathrm{D}$ & $E$ & $F$ & $\mathrm{G}$ & $\mathrm{H}$ & I & $\mathrm{J}$ & $\mathrm{K}$ & $L$ & $\mathrm{M}$ & $\mathrm{N}$ & 0 & $P$ & $Q$ & $R$ & $S$ & $\mathrm{~T}$ \\
\hline
\end{tabular}

2 
Table 2 (on next page)

Software, sub-commands, and parameters used for pyani, sourmash, FastANI, and LINflow. 
1

\begin{tabular}{|l|l|l|}
\hline Software & Version & Parameters \\
\hline pyani $^{1}$ & 0.2 .9 & $-\mathrm{m}$ ANIb -worker 100 \\
\hline sourmash & 2.0 .0 & $-\mathrm{k} 21,51-\mathrm{n} 2000$ \\
\hline FastANI & 1.2 & $-\mathrm{k} 16$-t 1 \\
\hline
\end{tabular}

$2{ }^{1}$ Multiprocessing was enabled.

3 


\section{Table $\mathbf{3}$ (on next page)}

Runtime of each software and sub-command used to analyze data sets A, B, C and D. 
1

\begin{tabular}{|c|c|c|c|c|c|c|}
\hline Data set & $\begin{array}{l}\text { No. of } \\
\text { genomes }\end{array}$ & pyani $^{1}$ & $\begin{array}{l}\text { sourmash } \\
\text { compute }\end{array}$ & $\begin{array}{l}\text { sourmash } \\
\text { compare }\end{array}$ & FastANI $^{1}$ & LINflow \\
\hline $\mathrm{A}$ & 247 & $60862 \mathrm{~m} \mathrm{6s}$ & $15 \mathrm{~m} \mathrm{3s}$ & $14 \mathrm{~s}$ & $401 \mathrm{~m} 18 \mathrm{~s}$ & $829 \mathrm{~m} \mathrm{39s}$ \\
\hline B & 43 & $2107 \mathrm{~m} \mathrm{18s}$ & $2 \mathrm{~m} \mathrm{34s}$ & $2 s$ & $17 \mathrm{~m} \mathrm{49s}$ & $105 \mathrm{~m} \mathrm{50s}$ \\
\hline $\mathrm{C}$ & 140 & $7274 \mathrm{~m} \mathrm{18s}$ & $7 \mathrm{~m} \mathrm{43s}$ & $8 \mathrm{~s}$ & $86 \mathrm{~m} \mathrm{12s}$ & $171 \mathrm{~m} \mathrm{38s}$ \\
\hline $\mathrm{D}$ & 55 & $3292 \mathrm{~m} \mathrm{37s}$ & $1 \mathrm{~m} \mathrm{41s}$ & $3 \mathrm{~s}$ & $13 \mathrm{~m} 25 \mathrm{~s}$ & $22 \mathrm{~m} \mathrm{58s}$ \\
\hline
\end{tabular}

$2{ }^{1}$ Total CPU time is listed for pyani and FastANI although multiprocessing was enabled.

3 


\section{Table 4 (on next page)}

Memory usage (GB) of each software and sub-command used to analyze data sets $A, B$, $C$ and D. 
1

\begin{tabular}{|l|l|l|l|l|l|}
\hline Data set & pyani & $\begin{array}{l}\text { sourmash } \\
\text { compute }\end{array}$ & $\begin{array}{l}\text { sourmash } \\
\text { compare }\end{array}$ & FastANI & LINflow \\
\hline A & 5.8 & 0.05 & 1.4 & 4.6 & 0.6 \\
\hline B & 1.2 & 0.04 & 0.1 & 0.5 & 0.3 \\
\hline C & 5.7 & 0.05 & 1.2 & 2.2 & 0.3 \\
\hline D & 1.3 & 0.05 & 0.1 & 0.4 & 0.3 \\
\hline
\end{tabular}

2 


\section{Table 5 (on next page)}

Runtime of each software and sub-command used to add a single new genome to the analyzed data set $A$. 
1

\begin{tabular}{|l|l|}
\hline Software and sub-command & CPU time \\
\hline pyani & $258 \mathrm{~m} \mathrm{23s}$ \\
\hline sourmash compute & $0 \mathrm{~m} \mathrm{4s}$ \\
\hline sourmash search & $0 \mathrm{~m} \mathrm{11s}$ \\
\hline FastANI & $4 \mathrm{~m} \mathrm{4s}$ \\
\hline LINflow & $3 \mathrm{~m} \mathrm{36s}$ \\
\hline
\end{tabular}

2 
Figure 1

Workflow of LINflow.

The flowchart of the LIN assignment algorithm used in LINflow. 


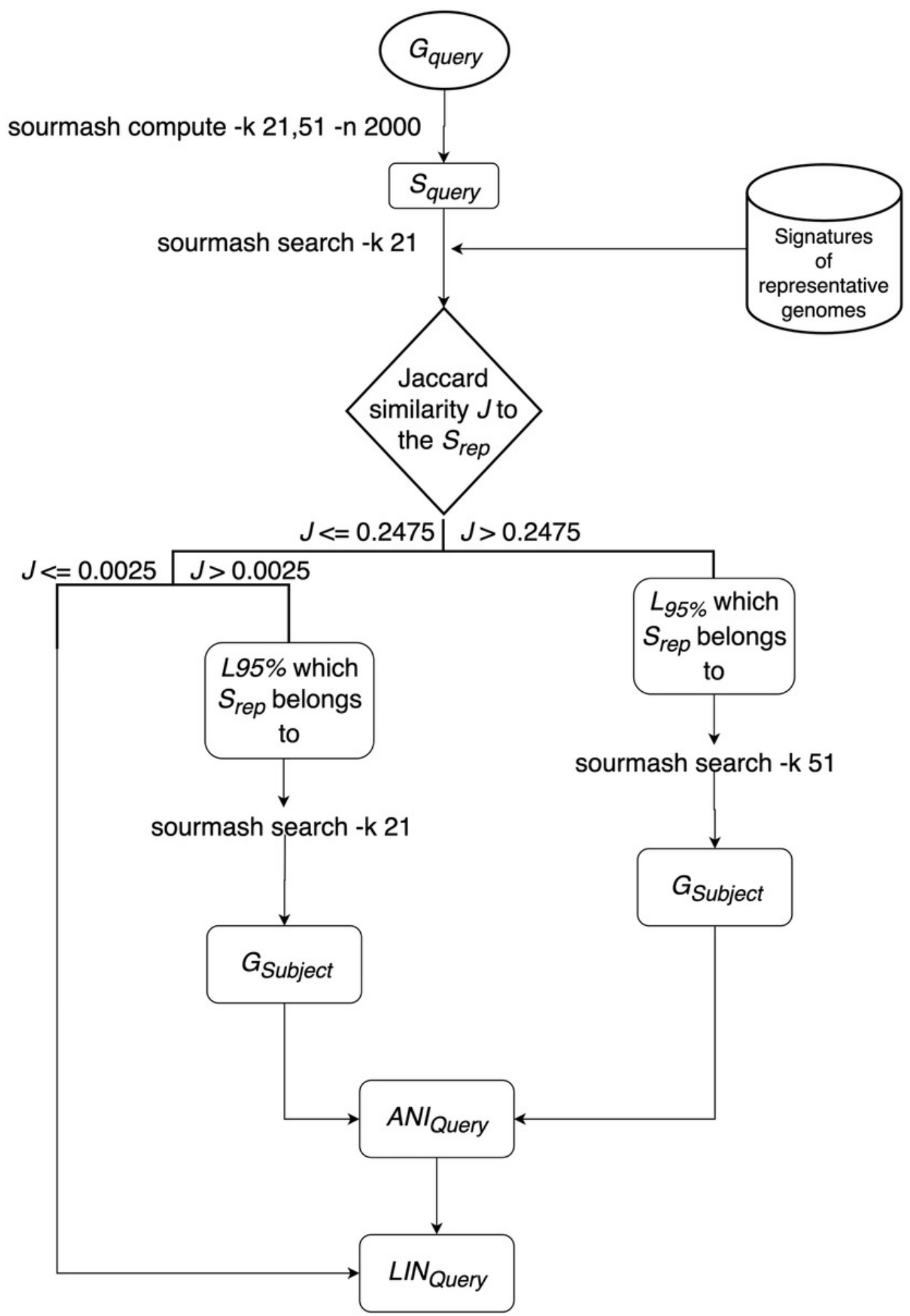


Figure 2

Database schema used by LINflow.

The relational database connects tables with primary and foreign keys. Connections between tables are represented by arrows. 


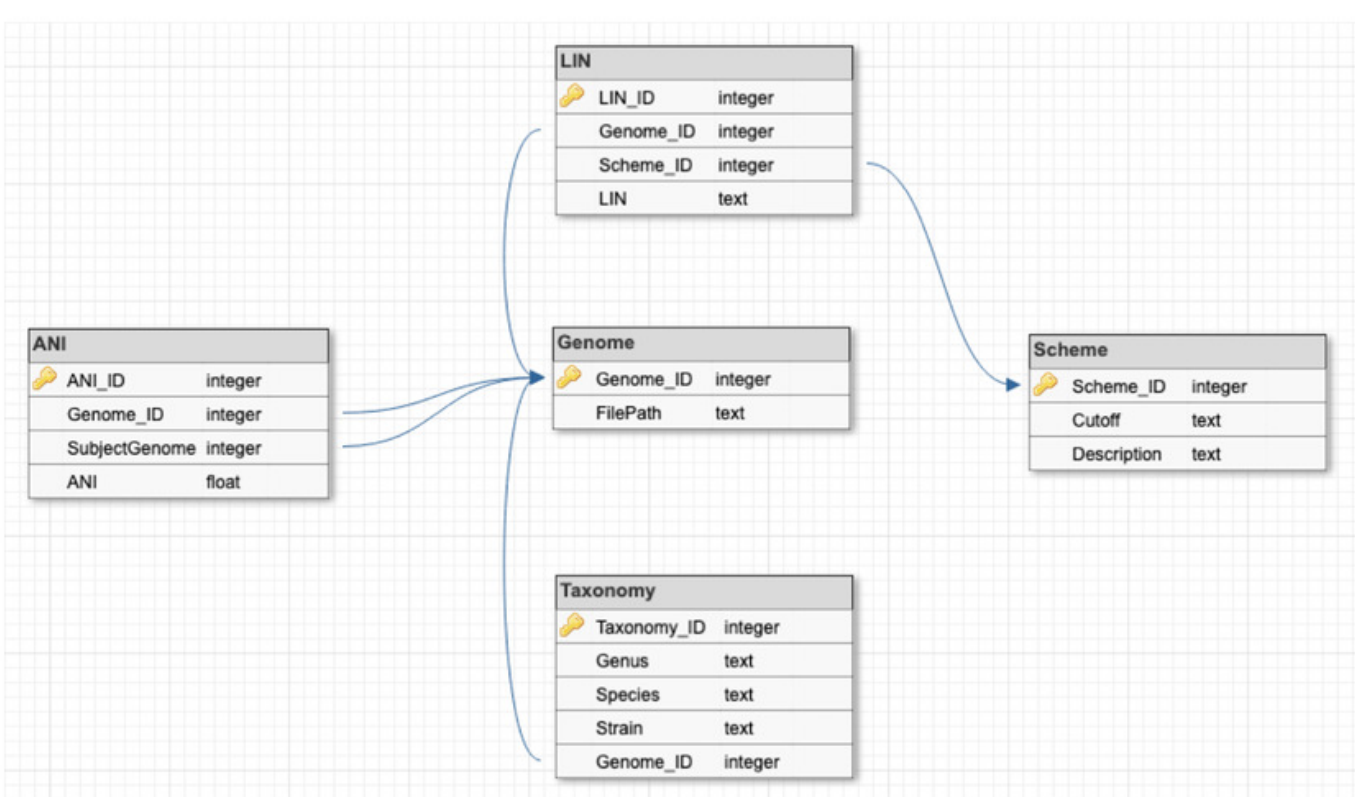




\section{Figure 3}

Similarity matrices obtained by LINflow, FastANI, sourmash and pyani.

Heatmaps based on hierarchical clustering using the complete linkage method using the similarity matrices obtained with pyani (A), FastANI (B), LINflow (C), Sourmash $k=21$ (D), and $\mathrm{k}=51(\mathrm{E})$ for dataset $\mathrm{A}$. Cluster 1 corresponds to $P$. aeruginosa, cluster 2 represents $P$. chlororaphis, clusters 3,4 , and 5 are different phylogroups within the $P$. syringae species complex and related genomes. The same figure showing strain names is included as Supplementary Figures 1 through 5 (corresponding to panels A through E). See Supplementary Figures 6 through 20 for heatmaps of datasets B, C, and D. 

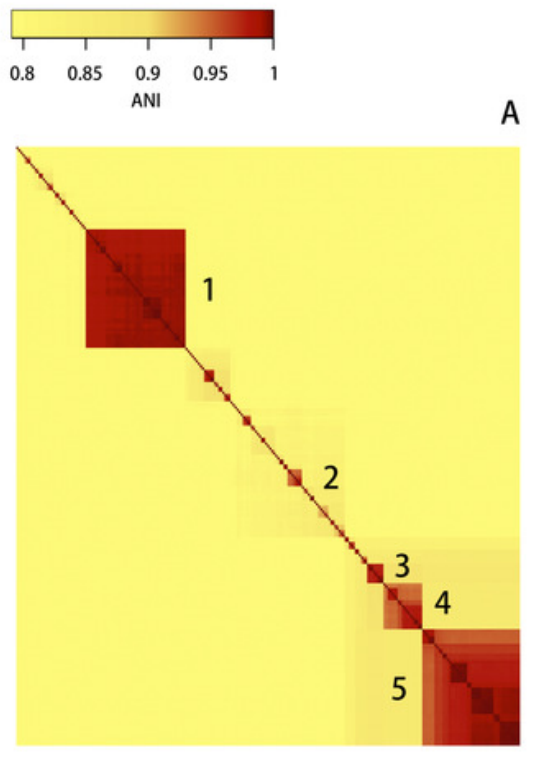

C
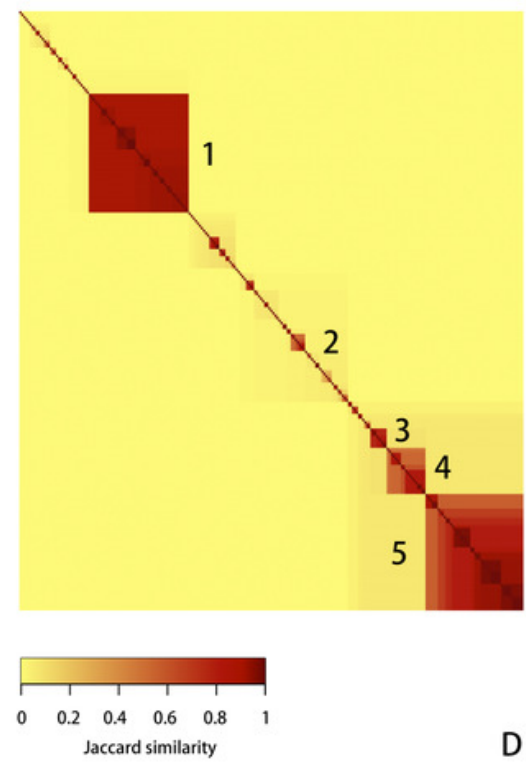

D

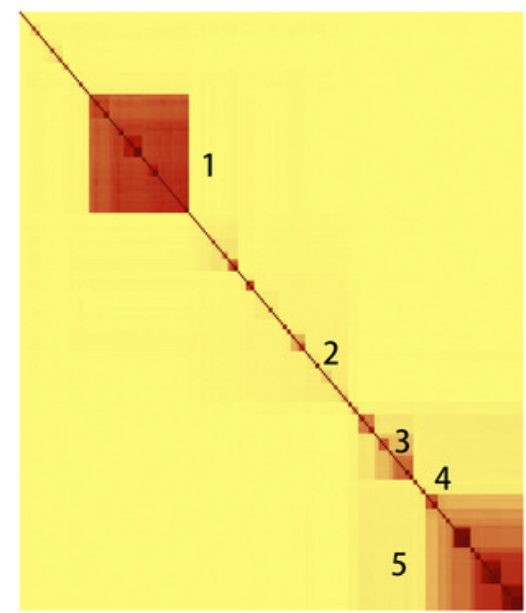

A

\section{,}

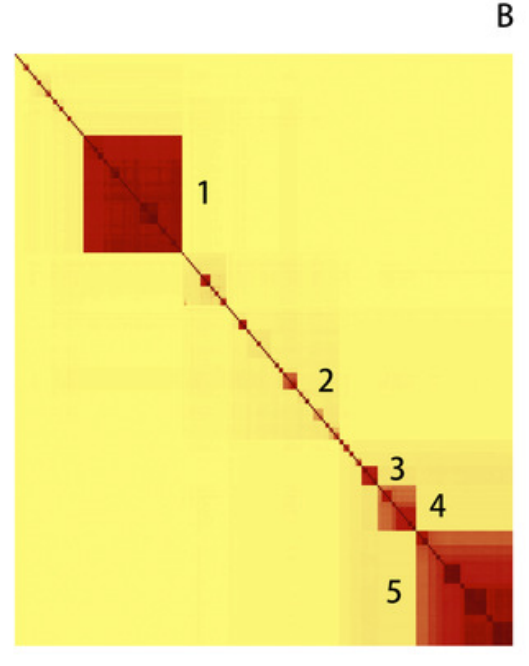

B 


\section{Figure 4}

Comparison of similarity matrices obtained by LINflow, FastANI, sourmash and pyani Heatmaps showing Pearson correlation coefficients based on the Mantel test performed between the similarity matrices obtained with pyANI, sourmash, FastANI, and LINflow for datasets $A, B, C$, and D. 
Data set $A$

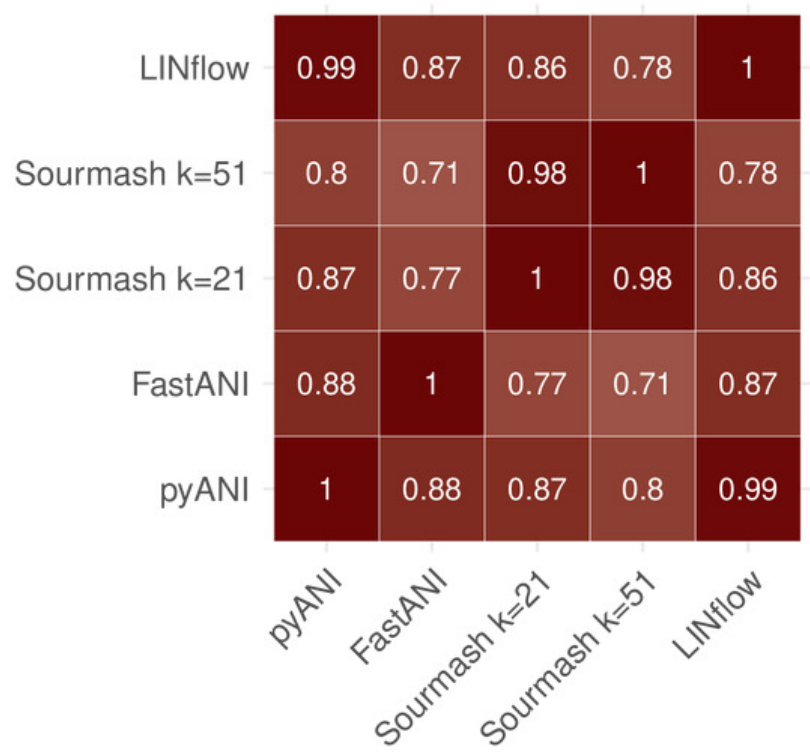

Data set B

\begin{tabular}{|c|c|c|c|c|c|c|c|}
\hline LINflow_300k & 0.82 & 0.82 & 0.84 & 0.87 & 0.87 & 1 & 1 \\
\hline LINflow_3k & 0.82 & 0.81 & 0.83 & 0.86 & 0.85 & 1 & 1 \\
\hline LINflow_300 & 0.78 & 0.8 & 0.83 & 0.85 & 1 & 0.85 & 0.87 \\
\hline Sourmash $\mathrm{k}=51$ & 0.88 & 0.88 & 0.99 & 1 & 0.85 & 0.86 & 0.87 \\
\hline Sourmash $\mathrm{k}=21$ & 0.86 & 0.85 & 1 & 0.99 & 0.83 & 0.83 & 0.84 \\
\hline FastANI & 0.91 & 1 & 0.85 & 0.88 & 0.8 & 0.81 & 0.82 \\
\hline pyANI & 1 & 0.91 & 0.86 & 0.88 & 0.78 & 0.82 & 0.82 \\
\hline
\end{tabular}

Data set D

Data set C

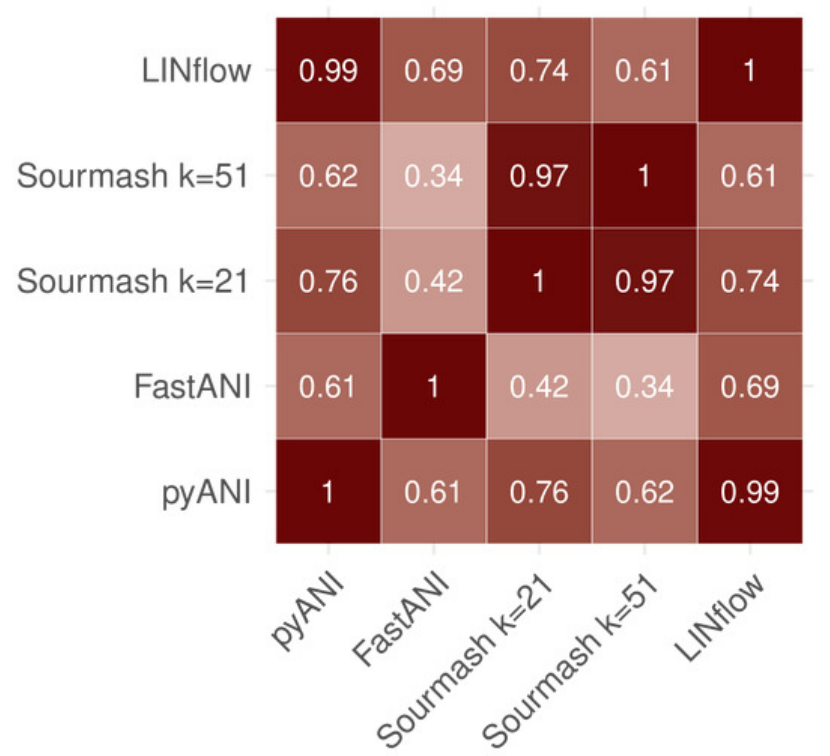

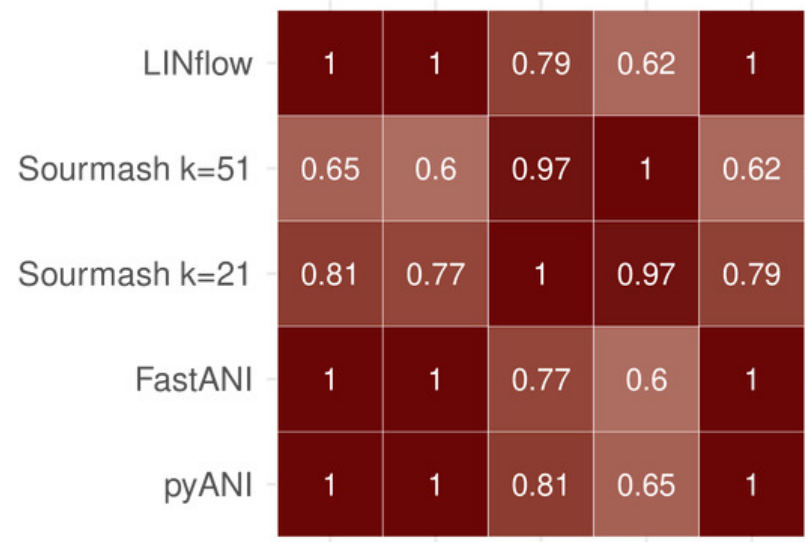

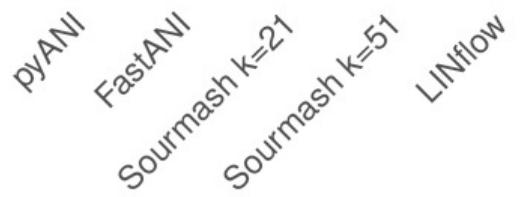




\section{Figure 5}

Correlation between the ANI results obtained with LINflow and FastANI and the ANI results obtained with pyani (using the BLAST option) for datasets A through D.

A Plot showing the ANI values computed by LINflow (in blue) and FastANI (in red) on the $Y$ axis for ANI results obtained with pyani (X axis) for all pairwise genome comparisons in datasets A through D. Pearson correlation coefficients for FastANI versus pyani and LINflow versus pyani results and all other tool comparison results are shown in Figure 4. B Plot showing the differences between the ANI values computed by FastANI and LINflow compared to the pyani ANI values for all pairwise ANI values for datasets A though D. 


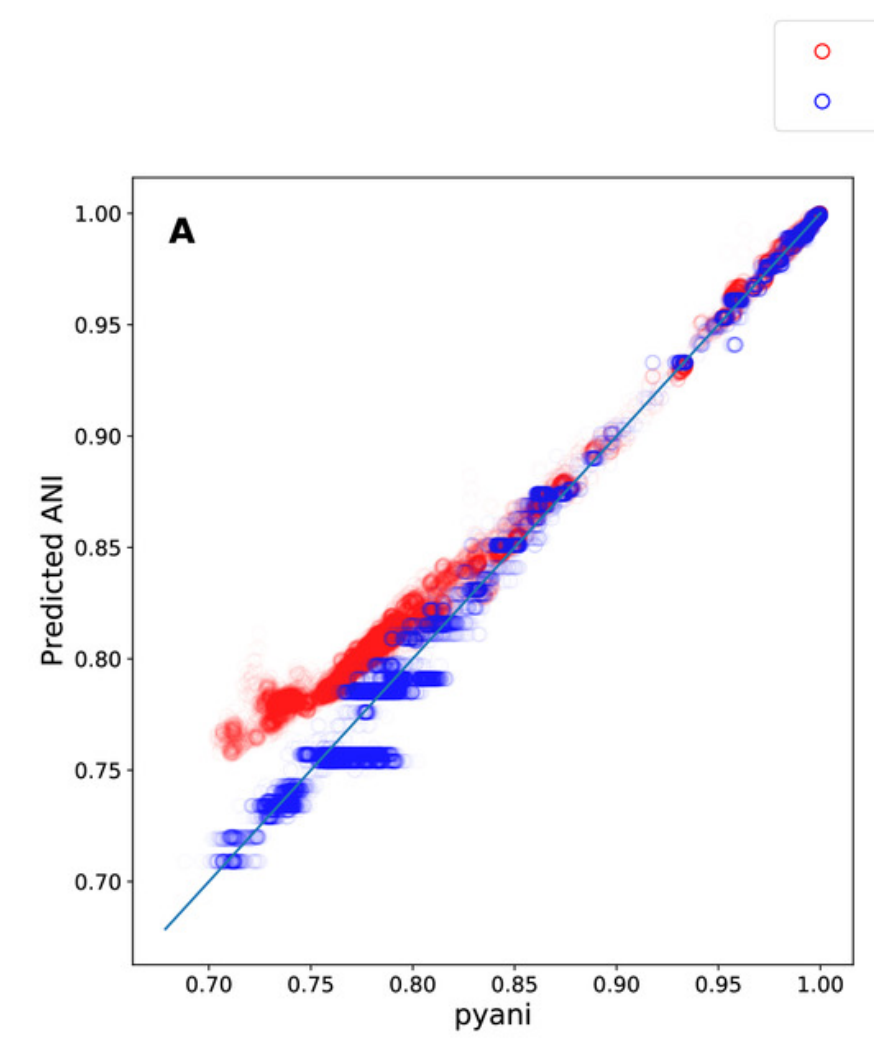

FastANI

- LINflow

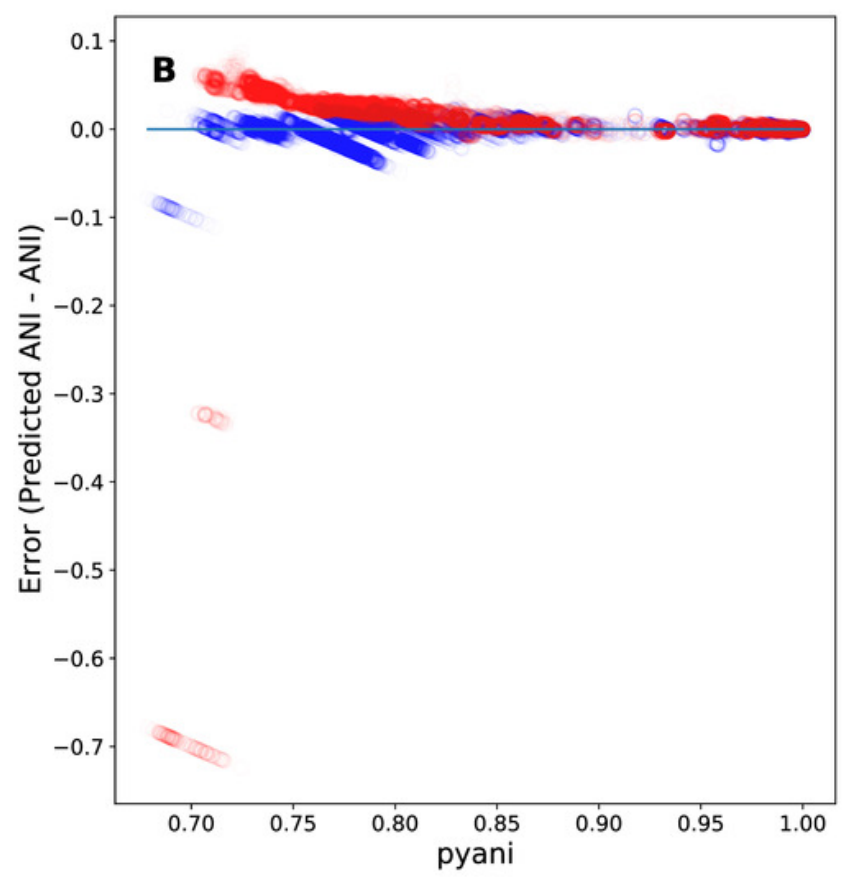

